\title{
A Food Monitoring System Based on Bluetooth Low Energy and Internet of Things
}

\author{
Mr.A.Venkatesh ${ }^{1}$, T.Saravanakumar ${ }^{2}$, S.Vairamsrinivasan ${ }^{3}$,A.Vigneshwar ${ }^{4}$, \\ M.Santhosh Kumar ${ }^{5}$ \\ ${ }^{1,}$ Assistant Professor, Department of ECE, Hindusthan Institute of Technology \\ ${ }^{2,3,4,5}$ Final Year Students, Hindusthan Institute of Technology, Coimbatore
}

\begin{abstract}
A Food Monitoring System Based on Bluetooth Low Energy(BLE) and Internet of Things(IoT)have provided a plethora of benefits to a variety of commercial industries, including the agricultural, biomedical, cosmetics, environmental, food manufacturing, military, pharmaceutical, regulatory,and various scientific research fields. In this System there exists a gas sensor which is used to detect the gases evolved from the food, temperature sensor to detect the temperature of the food storage area and the humidity sensor to check the humidity. The collected data from the test samples is transferred to the application through Bluetooth using Bluetooth Low Energy(BLE) or Internet of Things(IoT) based on the range of the server. The system uses a GSM/GPRS public wireless network for remote data transfer The combination of internet of things technology, GSM / GPRS public wireless network technology and Internet significantly reduces the cost of the system, with leading limitless scope of tracking recognition, which enhances the comprehensive performance of the system greatly. Advances have improved productattributes, uniformity, and consistency as a result of increases in quality control capabilities afforded by A Food monitoring system based on Bluetooth Low Energy(BLE) and Internet of Things of all phases of industrial manufacturing processes. This paper is a review of some of the more important and modern applications that have been of greatest benefit to the humankind.
\end{abstract}

Keywords: Bluetooth Low Energy(BLE); Internet of Things (IoT); Food safety; Quality tracing.

\section{INTRODUCTION}

The resolution of the Uruguay Round of the GeneralAgreement on Tariffs and Trade (GATT) in 1995 ,recognized public health risk as the only basis for restrictions of international trade in food.In recent years, food safety incidents have occurred frequently, such as mad cow disease, avian influenza, foot-andmouth disease, swine hammer infectious diseases and food poisoning, which have not only led to huge economic losses on a global scale, but also seriously endangered people's health across the world. Thus, food safety management and traceability becomes particularly important. Since the existing methods can't very well solve the problem of food traceability and evaluation, most people have to make choice by themselves, for most of the agricultural products, especially meat, milk, seafood, fruit, and so on involve the interests of the multitude. It is an important subject that presses for finding an effective method of tracking, management, tracing and evaluating to ensure food safety.In the current food industry, there are three steps between food production and final consumption, including processing, transportation and stocking. If problems occur at any point in food-handling sequences, it is possible to expose food in the state of insecurity, such as accessing to the source improperly that causes food spoilage. Excellent food safety system can provide seller with advice of delivery order and consumers with food quality and safety report, and manufacturers with market analysis report, etc. Food quality monitoring system based on Bluetooth low energy and internet of things provides an effective technology to solve this problem, which can be used to achieve food safety management, tracing and evaluating BLE and Internet of things is a non-contact automatic identification technology, which automatically identify target and get the relevant data through radio frequency signals and combine effective database system with network system to realize tracking items worldwide and information sharing. Compared with traditional bar code technology, internet of things technology features work identification without manual intervention, bulk read over a long distance, lowrequirements for environment, long service life, encrypting information stored in the data, etc. Its application will bring fundamental change to food safety tracing management.

According to the characteristics of the BLE and internet of things technology, this paper puts forward the food safety traceability system based on internet of things technology. Starting from varieties breeding to production processing, it labels records with an electronic tag, including all the information in the process of circulation: transportation, packaging, 
repackaging, and marketing. The information on food supply chain can be automatically obtained and target object are identified easily with data reading and writing at any time and places in the process of circulation, which helps to judge food quality. On the basis of analyzing business process, the use of internet of things technology in each link of food circulation is discussed, among which business model, system construction and implementation of the strategy are centered on.

\section{LITERATURE SURVEY}

$>$ On 16 July 2013, at least 23 students died and dozens more fell ill at a primary school in the village of DharmashatiGandaman in the Saran district of the Indian state of Bihar after eating a Midday Meal contaminated with pesticide.The food material for midday meals was stored at the house of the headmistress as the school did not have sufficient infrastructure.

$>$ In the major markets of the cities and towns in India, Vegetables and fruits gets wasted due to the temperature and humidity changes.

$>$ India is considered to be a major producer of milk and milkproducts, during export it is necessary to monitor the products.

$>$ In markets green leaves are gets wasted due to the fluctuations in temperature and humidity.

$>$ Fruits like Apple, Oranges and vegetables like Carrot are produced in hill areas. These products must be safely distributed to other region. So, it must be monitored during the transportation.

\section{EXSISTING WORK}

1. Kong Xiansheng and Sun Jingintroduces Near Field Communication and comprises a $\mathrm{pH}$ sensor and an external interrogator thatinteract to provide information on the quality of food products. The $\mathrm{pH}$ sensor is further divided into electrodes and resonant circuit. The electrodes of the sensor are coated with hydrogel; a hydrophilic polymer which acts as an electrolytic solution where the electrodes interact. The resonant circuit consists of an inductive coil and sensor electronics.

2. Vassiliss.Kodogiannis\&AbeerAlsahejari-July 2016 introduce A Fuzzy-wavelet neural network model for the detection of meat spoilage using an electronic nose. This provides the information of the food based on the temperature and humidity using temperature and humidity sensor.

3. Phurnvirachongthanasphisut, Tharaseesaared,Teer akiratkerdcharoen-Aug 2015 introduce Monitoring of microbial canned food spoilage and contamination based on e-nose for smart home.This system Senses ammonia in canned foods by the principal of component analysis(PCA) and it produces the current status of the canned foods.

4. SharmithaBhadra,DouglasJ.Thomson-July 2015 present a new $\mathrm{CO} 2$ sensor based on a hydrogel $\mathrm{pH}$-sensitive electrode pair. This sensor provides a direct voltage measurement depending on the $\mathrm{CO} 2$ concentration in the surrounding environment. We have integrated this sensor into a chipless near-field coupled RFID tag.

\section{PROPOSED METHOD}

In this system Bluetooth Low Energy technology is used to transmit the data from the microcontroller to the server. In this case BLE uses a very low energy of only less than $15 \mathrm{~mA}$ whereas the classic Bluetooth technology can consume the power consumption up to $30 \mathrm{~mA}$. The size of the system is small when compared to the existing systems.

\section{1. nRF52832 MICROCONTROLLER:}

The nRF52832 incorporates a powerful Cortex-M4F processor enabling the most demanding applications with complex arithmetic requirements to be realized in a single chip solution. The IC supports DSP instructions, a Floating Point Unit (FPU), singlecycle multiply and accumulate, and hardware divide for energy-efficient process of computationally complex operations. The $2.4 \mathrm{GHz}$ radio supports multiple protocols including Bluetooth low energy, ANT and $2.4 \mathrm{GHz}$ proprietary. The radio has high definition RSSI and highly automated functionality, including EasyDMA for direct memory access during packet send and retrieve. Nordic provides protocol stacks for Bluetooth low energy.The nRF52832 is an extremely power efficient device that can run from a supply between $1.7 \mathrm{~V}$ and $3.6 \mathrm{~V}$. All individual peripherals and clocks offer complete flexibility of power down when not required for taskoperation thus minimizing power consumption.

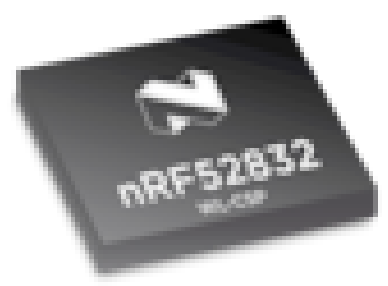

Fig1:Nrf52832 microcontroller 


\section{SENSORS:}

- VOC SENSOR:

VOC sensors use a bright ultraviolet light source to knock electrons out of the VOC molecule; the electrons are measured and their flow indicates that VOC molecules are present at the sensor. Each specific type of VOC molecule has an 'ionization potential' (IP) value that represents the amount of energy necessary to liberate an electron; this value is measured in 'electron volts', or eV. PID sensors have a specified level of energy, also measured in $\mathrm{eV}$, and in general, any compound with an IP value less than the sensor's eV rating will be ionized and detected.

\section{- DHT11 SENSOR:}

DHT11 uses a capacitive humidity sensor and a thermistor to measure a surrounding air.The values are triggered by using clock pulse whenever it is needed.Hence the input from the sensor is send to the microcontroller by using the optocoupler circuit. The optocoupler circuit can avoid the damages to the electronic circuit when there exist an voltage fluctuations.

\section{BLE TECHNOLOGY}

Bluetooth Smart technology operates in the same spectrum range (the $2.400-2.4835 \mathrm{GHz}$ ISM band) as Classic Bluetooth technology, but uses a different set of channels. Instead of the Classic Bluetooth 79 1-MHz channels, Bluetooth Smart has $402-\mathrm{MHz}$ channels. Within a channel, data is transmitted using Gaussian frequency shift modulation, similar to Classic Bluetooth's Basic Rate scheme. The bit rate is $1 \mathrm{Mbit} / \mathrm{s}$, and the maximum transmit power is $10 \mathrm{~mW}$.

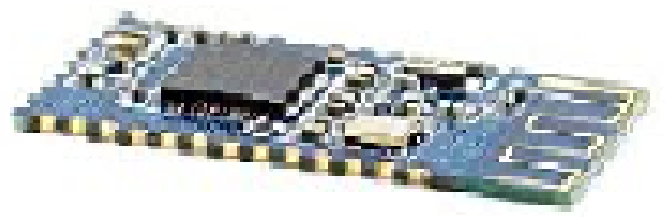

Fig1:BLE technology

\section{THINGSPEAK API:}

"ThingSpeak is an open source Internet of Things (IoT) application and API to store and retrieve data from things using the HTTP protocol over the Internet or via a Local Area Network. ThingSpeak enables the creation of sensor logging applications, location tracking applications, and a social network of things with status updates.In this system we can use the XAMPP server to send and receive the data. The graphical format of the food information is viewed in the graphical format using the thingspeak web application.

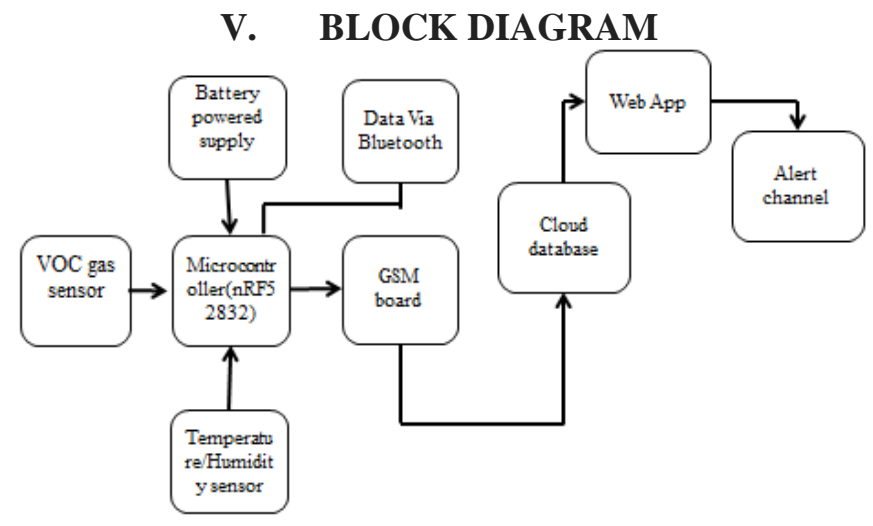

\section{RESULTS AND DISCUSSION}

A food monitoring system based on Bluetooth low energy and Internet of things have been utilized in a wide and diverse range of applications such as odor analysis, qualitycontrol in the food industry, environmental protection detection, public health, explosives detection and spaceflight applications to improve effectiveness, efficiency and safety. Due to the complexity of biological olfaction, the artificial olfactory system has non-linearity characteristics. Due to the differences ofselectivity, sensitivity and specificity of sensors, the optimal features of sensors are various. Usually, the same features are extracted from different sensors with the special methods which we reviewed above. However, it is possible that the features are not optimal for each sensor and better performance can be obtained if different features are extracted and fused for pattern recognition with different feature methods for different sensors. 

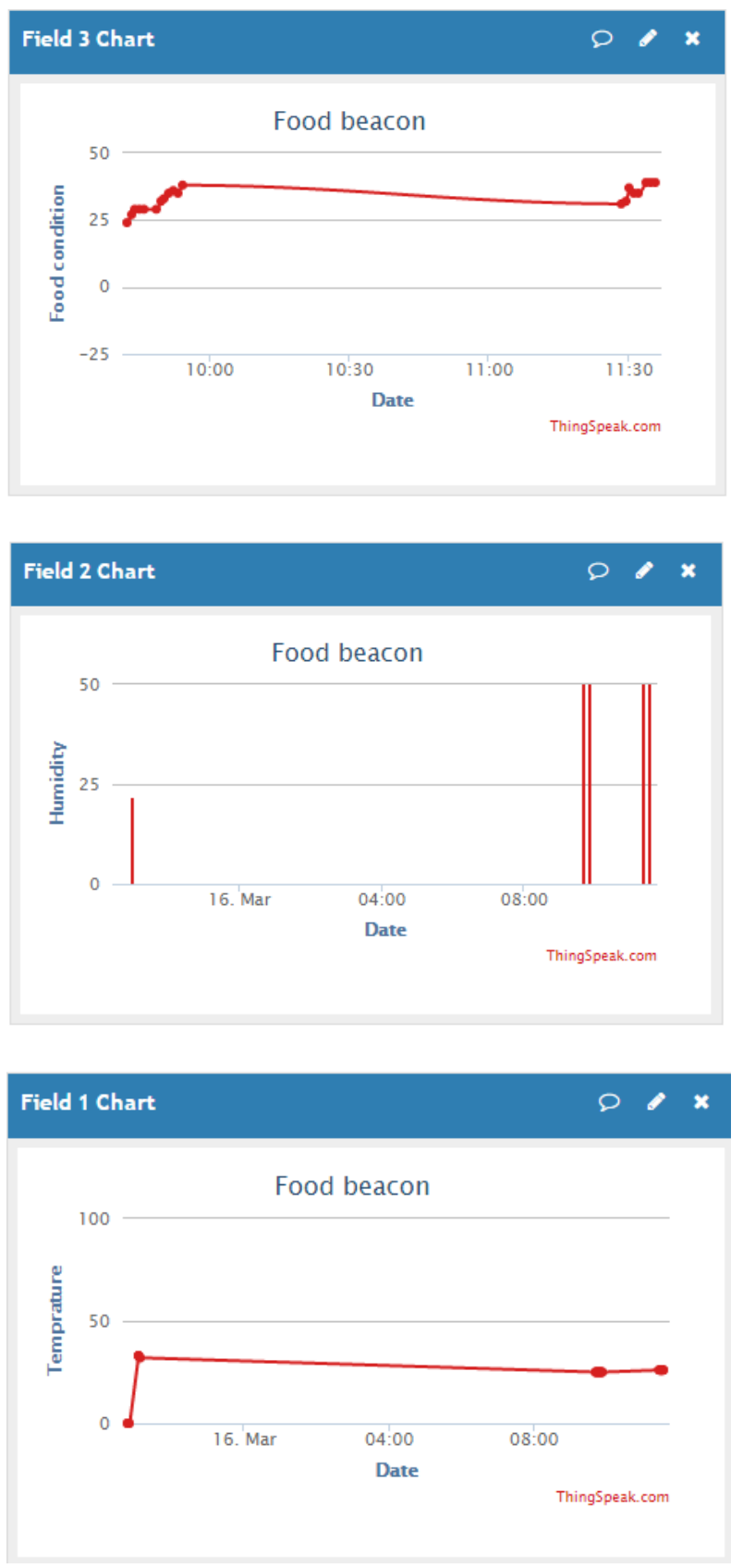

\section{CONCLUSION}

With a vast amount of literature now available to demonstrate the theoretical and practical feasibilityof using food monitoring system based on BLE technology and IoT in many diverse applications, the trick now will be to go the extra distance to beginto fine-tune these technologies for many practical and specific applications. This will require cooperativeefforts and informative exchanges between researchers and practitioners. Once these difficulties and logisticsare resolved, food monitoring system based on BLE and IoT devices should be capable of solving many problems and serving many of the future needs of the industries that have yet to be discovered.

\section{REFERENCES}

[1] LiuChuanju. Research on the digitized production model under the network environment[J], Journal ofNetworks, 2011,12-3 :160-163;

[2] YuShanshan, based on NET-business supply chain management system design andrealizationmicrocomputer, 2011,12-3 :168-170;

[3] LiuChuanju. the cluster greenhouse optimization design of the remote monitoring system under thenetworkenvironment.Journal of Multimedia .2012 (12): P82-84; 
[4] LiMinzhe, Sun Junmao, Cao Xinming, the status quo, debate and suggestions of the safety of agriculturalproducts in China. China Agricultural Science and Technology, 2012, 5 (3) :68-71

[5] Zhangbing Wen, HaoZhenghong. Safety of agricultural products in China.food safety topics, 2000.

[6] Sanford, V., Pervasive Computing Goes the Last Hundred Feet wit internet of things Systems, IEEE CS andIEEE ComSoc, AprilJune, 2013.

[7] Wind, G. Creating Business Intelligence from What Were Black Holes of Informationand Data, internet of thingsWorld, Sydney, 2011.

[8] Ngai E W $\mathrm{T}$ et al. internet of things Research: An Academic Literature Review(1995-2005) and Future

ResearchDirections $[\mathbf{J}]$.International Journal of Production Economics, 2012, 112( 2) : $510-520$.

[9] MKarkkainen. Increasing Efficiency in the Supply Chain for Short Shelf life Goods Using internet of thingsTagging $[\mathrm{J}]$. Journal of Multimedia, 2011, 31( 10) : $529-536$.

[10] Finkenzeller, K.: internet of things Handbuch: Grundlagen und praktischeAnwendungeninduktiverFunkanla gen, Transponder und kontaktloserChipkarten. 4 Auflage, Hanser, München, 2010

[11] Jedermann, R.; Edmond, J.P.; Lang, W.: Shelf life prediction by intelligent internet of things. In Haasis, H.D.;Kreowski, H.J.; Scholz-Reiter, B. (Eds.): Dynamics in Logistics - First International Conference, LDIC 2011Bremen, Germany, August 2012Proceedings. Springer, Berlin, 231-238, May 2008

[12] Jedermann, R. und Lang, W.: TechnischeGrenzen des Einsatzes von UHF Identifikationssystemeninternet ofthings) imLebensmittelbereich. Technical Report SFB637-B6-07-3, University of Bremen, November 2011

[13] Jedermann, R.; Lang, W.: Semi-passive internet of things and beyond: steps towards automated quality tracingin the food chain. In: International Journal of Radio Frequency Identification Technology and Applications(IJRFITA), Vol. 1(2012)3, pp. $247-259$

[14] B G Anil Kumar,K C Bhagyalakshmi, K.Lavanya,K H Gowranga, "A bluetooth low energy based beacon system for smart short range surveillance".

[15] ShaarmisthaBhadra,DouglasJ.Thomson,Greg E.Bridges ,"Near-Field coupled RFID tag for carbon dioxide concentration sensing".

[16] PhunviraChongthanaphisut,TharaSeesaard,T eerakiatKerdcharoen,"Monitoring of microbial canned food spoilage \& contamination based on e-nose for smart home". 